\title{
Mérimée et le bon usage du savoir - La création à l'épreuve de la connaissance, sous la direction de Pierre Glaudes
}

\section{Silvia Lorusso}

\section{(2) OpenEdition Journals \\ Edizione digitale \\ URL: http://journals.openedition.org/studifrancesi/6704 \\ DOI: 10.4000/studifrancesi.6704 \\ ISSN: 2421-5856 \\ Editore \\ Rosenberg \& Sellier \\ Edizione cartacea \\ Data di pubblicazione: 1 septembre 2010 \\ Paginazione: 393 \\ ISSN: 0039-2944 \\ Notizia bibliografica digitale \\ Silvia Lorusso, «Mérimée et le bon usage du savoir - La création à l'épreuve de la connaissance, sous la direction de Pierre Glaudes», Studi Francesi [Online], 161 (LIV | II) | 2010, online dal 30 novembre 2015, consultato il 09 janvier 2021. URL: http://journals.openedition.org/studifrancesi/6704 ; DOI: https:// doi.org/10.4000/studifrancesi.6704}

Questo documento è stato generato automaticamente il 9 janvier 2021.

\section{cc) (†)}

Studi Francesi è distribuita con Licenza Creative Commons Attribuzione - Non commerciale - Non opere derivate 4.0 Internazionale. 


\title{
Mérimée et le bon usage du savoir - La création à l'épreuve de la connaissance, sous la direction de Pierre Glaudes
}

\author{
Silvia Lorusso
}

\section{NOTIZIA}

AA. VV., Mérimée et le bon usage du savoir - La création à l'épreuve de la connaissance, sous la direction de Pierre GLAUDES, Toulouse, P. U. du Mirail, 2008, pp. 244.

1 In occasione del bicentenario della nascita di Mérimée, questi atti dell'importante colloquio di Tolosa, che ha unito storici e letterati, approfondiscono diversi versanti delle personalità dello scrittore: personalità che, come osserva Pierre GLAUDES nell'Introduzione, nei suoi aspetti variegati e nei suoi multipli talenti (letterari, filosofici, etnografici, antiquari), presenta comunque vistose costanti. Prima fra tutte, una concezione del primitivo come violenza.

2 Vari saggi sono cosi dedicati alla carriera di Mérimée in quanto Ispettore dei Monumenti storici (Jean LECLANT) e, più in generale, alla sua attività di conoscitore $\mathrm{e}$ difensore dei monumenti antichi. Pierre PONTIER, riesumando le sue recensioni a History of Greece di Grote, mostra come la sua ricostruzione storica fosse animata anche da una prospettiva politica contemporanea: la critica della Repubblica del 1848 , attraverso l'elogio della democrazia ateniese e il consenso a Thiers, riconoscibile nei tratti di un nuovo Solone. Olivier PoIsson colloca la riflessione merimeiana sull'arte medievale all'interno del dibattito contemporaneo. Françoise BERCÉ illustra l'azione di Mérimée nei confronti dei monumenti romani del Sud della Francia e in particolare il suo impegno per il restauro dell'anfiteatro di Arles, per il quale lo Stato francese investì somme ingentissime. Mérimée sembra in sintonia più che con gli archeologi, con gli architetti del tempo. Jean-Marie PAILLE analizza l'interesse che lo scrittore ebbe per i resti romani e pre-romani della Gallia e in particolare ricorda la sua difesa dei 
«dolmens». Adeline GRAND-CLÉMENT mostra come il suo viaggio di cinque mesi in Grecia fosse insieme missione scientifica e pellegrinaggio iniziatico. Nonostante siano rimaste poche testimonianze di questo viaggio, Mérimée risulta, sulla scorta di Lenormant, un viaggiatore moderno, interessato ai monumenti greci, interpretati come culmine della bellezza, e però anche agli usi e costumi dei popoli. Catherine HUET-BRICHARD considera un'opera letteraria, La Guzla, ma in una prospettiva etnologica: giocando all'etnologo, lo scrittore dà voce ad alcuni temi ricorrenti nella sua opera narrativa, quale appunto la violenza del primitivo. Tale violenza, che sembra escludere ogni alternativa o cambiamento, si incarna in ruoli e situazioni archetipici: parricidio, fratelli nemici, tradimenti coniugali, vendicati sanguinosamente. Questa violenza, pur essendo estrema, non è mai anarchica: ma rispecchia una società nella quale il principio di autorità è potente e incontrastabile. François GÉAL analizza l'ultimo capitolo di Carmen dedicato ai Gitani e non solo discute delle sue fonti e della sua attendibilità scientifica, ma anche ne ribadisce il carattere tutt'altro che accessorio. Mérimée non distingue tra Rom (localizzati nei Balcani), Manouches (Nord Europa, Germania e Italia), Gitani (Penisola Iberica e Sud della Francia), bensì si interessa agli Zingari come insieme. Fu anche per questo che il IV capitolo fu criticato.

In ambito più propriamente letterario, Judith LYON-CAEN si interroga sui rapporti tra «connu» e «inconnu(e)» nei racconti merimeiani e distingue tre possibilità. La prima nel registro del «merveilleux»; la seconda, in cui «l'inconnu» è separato dal «connu» da uno scarto minimo - i misteri si trasformano in segreti; la terza in cui «l'inconnu» è abolito, come nel caso di racconti «anti-ermeneutici», quasi Mateo Falcone, Tamango, Arsène Guillot. Infine i saggi di Antonia FONYI e Pierre GLAUDES affrontano questioni cruciali per la definizione dell'arte e della personalità di Mérimée. Entrambi mettono l'accento su una contraddizione che anima quasi tutte le sue opere: da una parte una prospettiva evoluta, civile, razionalista che si esercita con orgoglio, ma che, tuttavia, non riesce a penetrare alcune dimensioni della realtà; dall'altra una prospettiva superstiziosa, arcaica, selvaggia, l'unica adeguata a fronteggiare e capire fenomeni che appartengono a stadi della culture e della vita psichica percepiti come primitivi. Questa realtà arcaica, violenta si presenta sempre come minacciosa e irresistibile. Carmen, Lokis, la statua di Venere mostrano tuttavia come tale dimensione abbia un'origine sessuale. A mio avviso, quindi, la possibilità di conoscenza, oggettiva o soggettiva, deve inevitabilmente confrontarsi col desiderio. E le stesse idee relative alla religione e alla superstizione non possono non fare i conti con essa. 